\title{
Transients in a Periodic Slab: Coupled Waves Approach
}

CHARLES ELACHI, MEMBER, IEEE, DWIGHT L. JAGGARD, STUDENT MEMBER, IEEE AND C. YEH, MEMBER, IEEE

\begin{abstract}
The reflection and transmission of rectangular and Gaussian pulses impinging on a periodically stratified slab are studied and a number of examples are illustrated and analyzed. The coupled waves approach is used to derive the reflection and transmission coefficient, then the fast Fourier transform is used to illustrate the transient responses.
\end{abstract}

\section{INTRODUCTION}

$\mathrm{M}$ OST OF THE WORK in the field of electromagnetic wave propagation in periodic structures has been directed toward continuous monochromatic waves [1]-[6]. However, it is of importance to study the transient response of these structures to finite pulses. In this paper we study the reflection and transmission of a rectangular pulse and a Gaussian pulse impinging on a periodic structure of finite length $l$. In our analysis, we consider the general case of transversely bounded structures where the coupled waves could be different modes and therefore have different longitudinal wave-vectors [6]. For simplicity, and with no loss of generality, the numerical examples will correspond to the special case of transversely unbounded structures. The coupled wave approach [7]-[9] is used, and we discuss briefly its limitations relative to the more general Floquet approach [1]-[5]. There is no limitation on the type of periodicity as long as the coupling coefficient $\chi$ is small enough to satisfy the coupled wave approach conditions.

\section{TRansfer Functions}

Let us consider a wave $F(z) U_{p}(x) e^{i \beta_{p} z}$ propagating in a transversely bounded periodic structure. The index $p$ represents the guided mode, $\beta_{p}$ is its longitudinal wave number, and $U_{p}(x)$ is its transverse dependence. This wave can be effectively coupled to a backward $q$ mode wave $B(z) U_{q}(x) e^{-i \beta_{q} z}$ if the phase matching condition

$$
\beta_{p}+\beta_{q}=\frac{2 \pi}{\Lambda}
$$

is satisfied, where $A$ is the period of the structure (Fig. 1). Let $\omega_{p q} /(2 \pi)$ be the frequency at which phase matching occurs. The coupled waves equations are well known to be [7]-[9]:

$$
\frac{d F}{d z}-i \delta_{p} F=i \chi_{p q} B
$$

Manuscript received July 29, 1974; revised December 30, 1974. This work was sponsored in part by NASA under Contract NAS7-100 and in part by NELC.

C. Elachi and D. L. Jaggard are with the California Institute of Technology, Jet Propulsion Laboratory, Pasadena, Calif. 91103.

C. Yeh is with the Electrical Sciences and Engineering Department, University of California, Los Angeles, Calif. 90024.

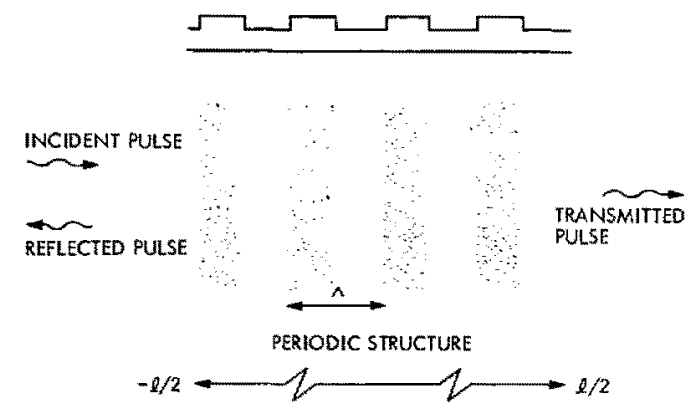

Fig. 1. Configuration. $l$ is thickness of periodic slab.

$$
\frac{d B}{d z}+i \delta_{q} B=-i \chi_{p q} F
$$

where $\chi_{p q}$ is the coupling coefficient which depends on the transverse functions $U_{p}(x)$ and $U_{q}(x)[6], \delta_{p}$ and $\delta_{q}$ are the wave numbers' mismatches and they are related to the frequency mismatch by

$$
\begin{aligned}
\delta_{p} & =\psi_{p} \Delta \omega=\left.\frac{\partial \beta_{p}}{\partial \omega}\right|_{\omega_{p q}} \Delta \omega \\
\delta_{q} & =\psi_{q} \Delta \omega=\left.\frac{\partial \beta_{q}}{\partial \omega}\right|_{\omega_{p q}} \Delta \omega \\
\Delta \omega & =\omega-\omega_{p q} \\
\omega & =\text { angular frequency. }
\end{aligned}
$$

$\psi_{p}$ and $\psi_{q}$ are the slopes of the dispersion curves of the guided waves. If $l$ is the length of the coupling region, then the longitudinal boundary conditions are (see Fig. 1)

$$
\begin{array}{ll}
F\left(-\frac{l}{2}\right)=1 & F\left(\frac{l}{2}\right)=T \\
B\left(-\frac{l}{2}\right)=R & B\left(\frac{l}{2}\right)=0
\end{array}
$$

where $T$ and $R$ are the transmission and reflection coefficients. The solution for the coupled wave equation (2) with the above boundary conditions gives

$$
\begin{aligned}
& R=\frac{i \chi_{p q}}{D \operatorname{coth} D l-i \frac{\delta_{p}+\delta_{q}}{2}} \\
& T=\frac{D e^{-i\left(\delta_{q}-\delta_{p}\right) l}}{D \cosh D l-i \frac{\delta_{p}+\delta_{q}}{2} \sinh D l}
\end{aligned}
$$




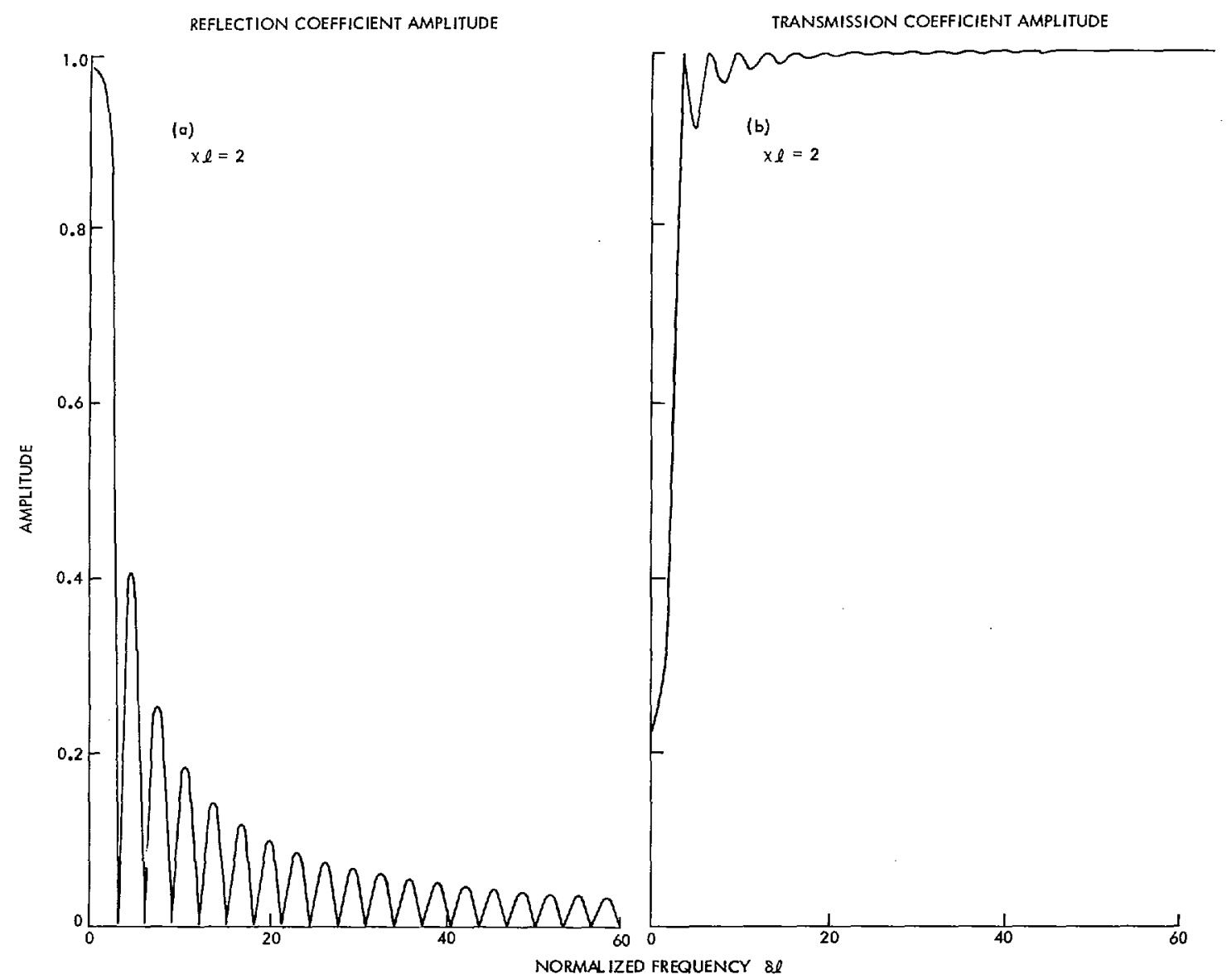

Fig. 2. Magnitude of reflection and transmission coefficients as function of normalized frequency mismatch: $\delta l=\Delta \omega l / c$ for fixed coupling: $\chi^{l}=2$.
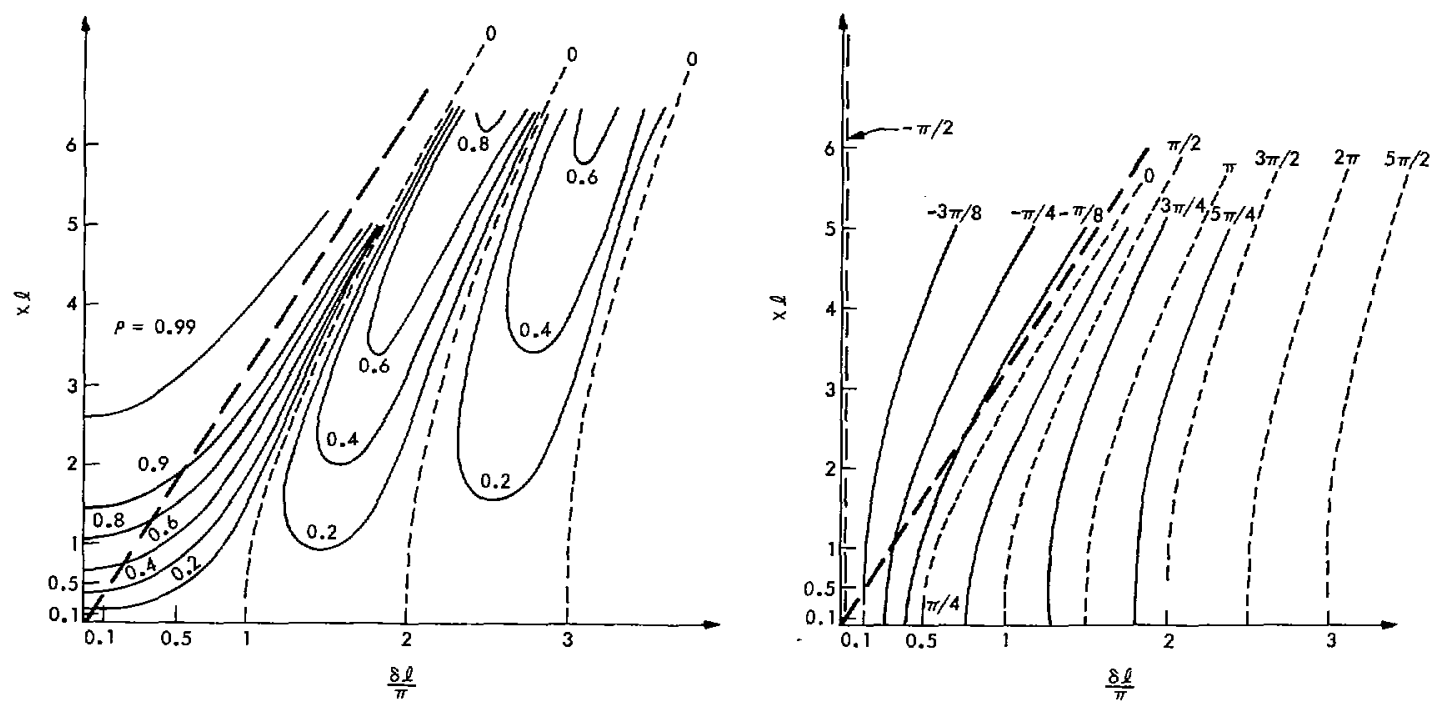

Fig. 3. Equimagnitude and equiphase curves of reflection coefficient as function of $\delta l$ and $\chi l$. Reflection curve in Fig. 2 corresponds to horizontal line $(\chi l=2)$ across graph. 


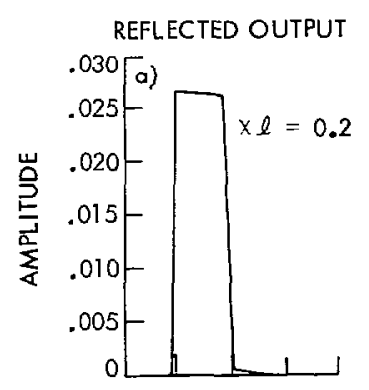

TRANSMITTED OUTPUT
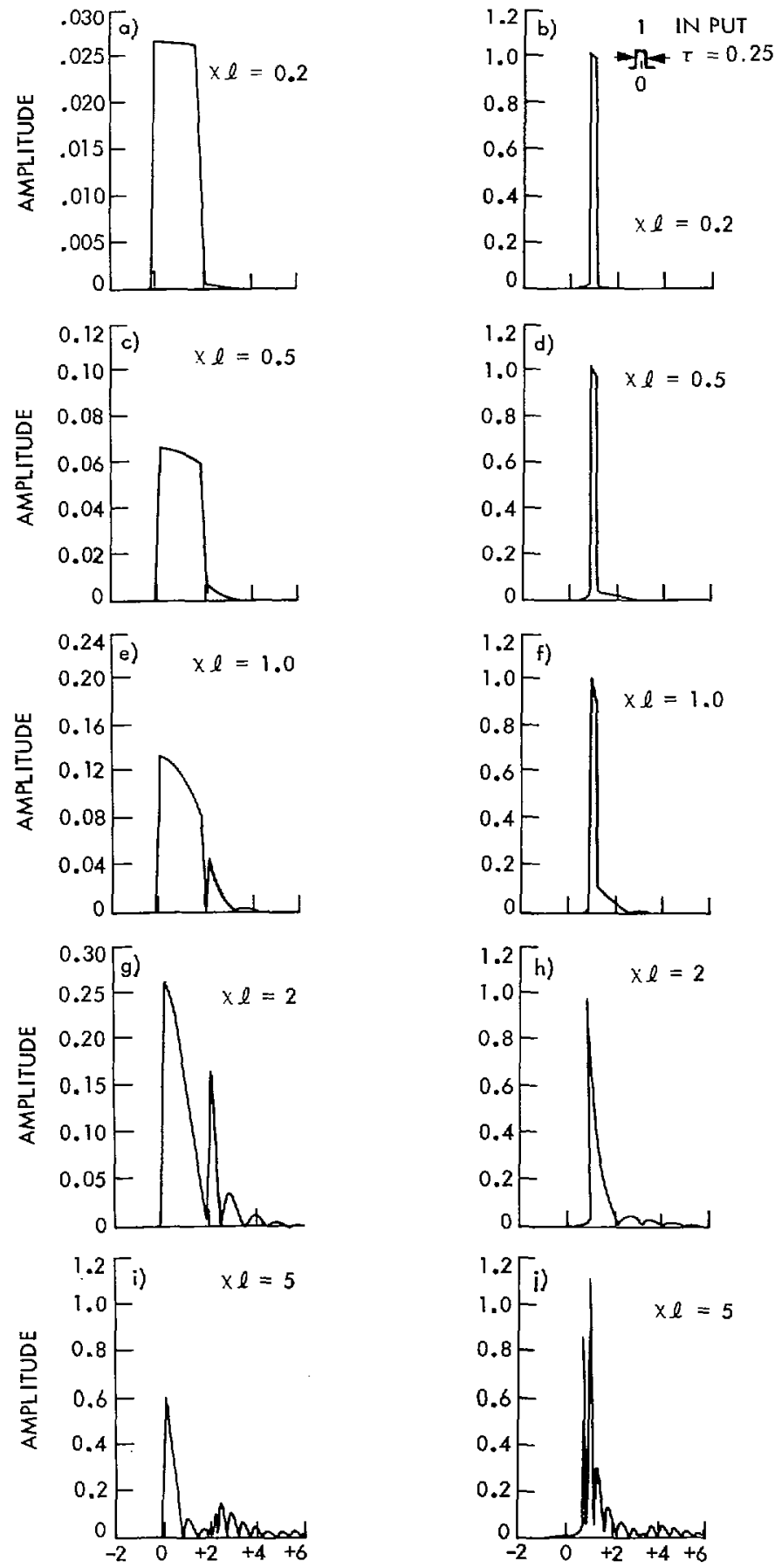

Fig. 4. Reflected and transmitted pulses for different values of coupling coefficient. Incident rectangular pulse has length of 0.25 time units and carrier frequency $\omega_{0}$ at Bragg condition. Remark difference in vertical scales.

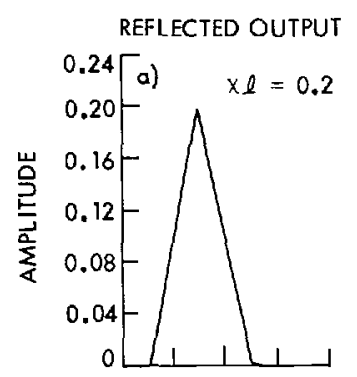

TRANSMITTED OUTPUT
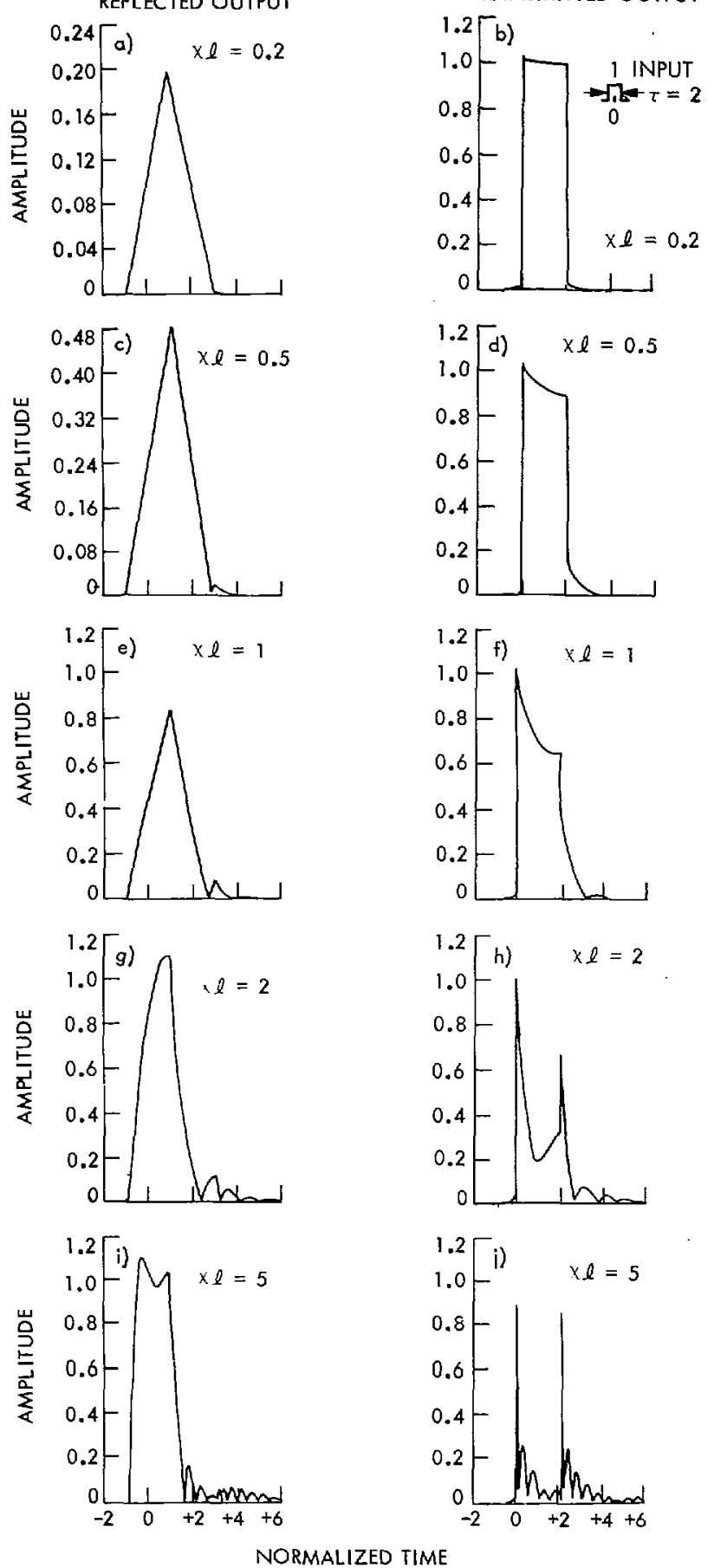

Fig. 5. Reflected and transmitted pulses for different values of coupling coefficient. Incident rectangular pulse has length of 2.0 time units and carrier frequency $\omega_{0}$ at Bragg condition. Remark difference in vertical scales. 

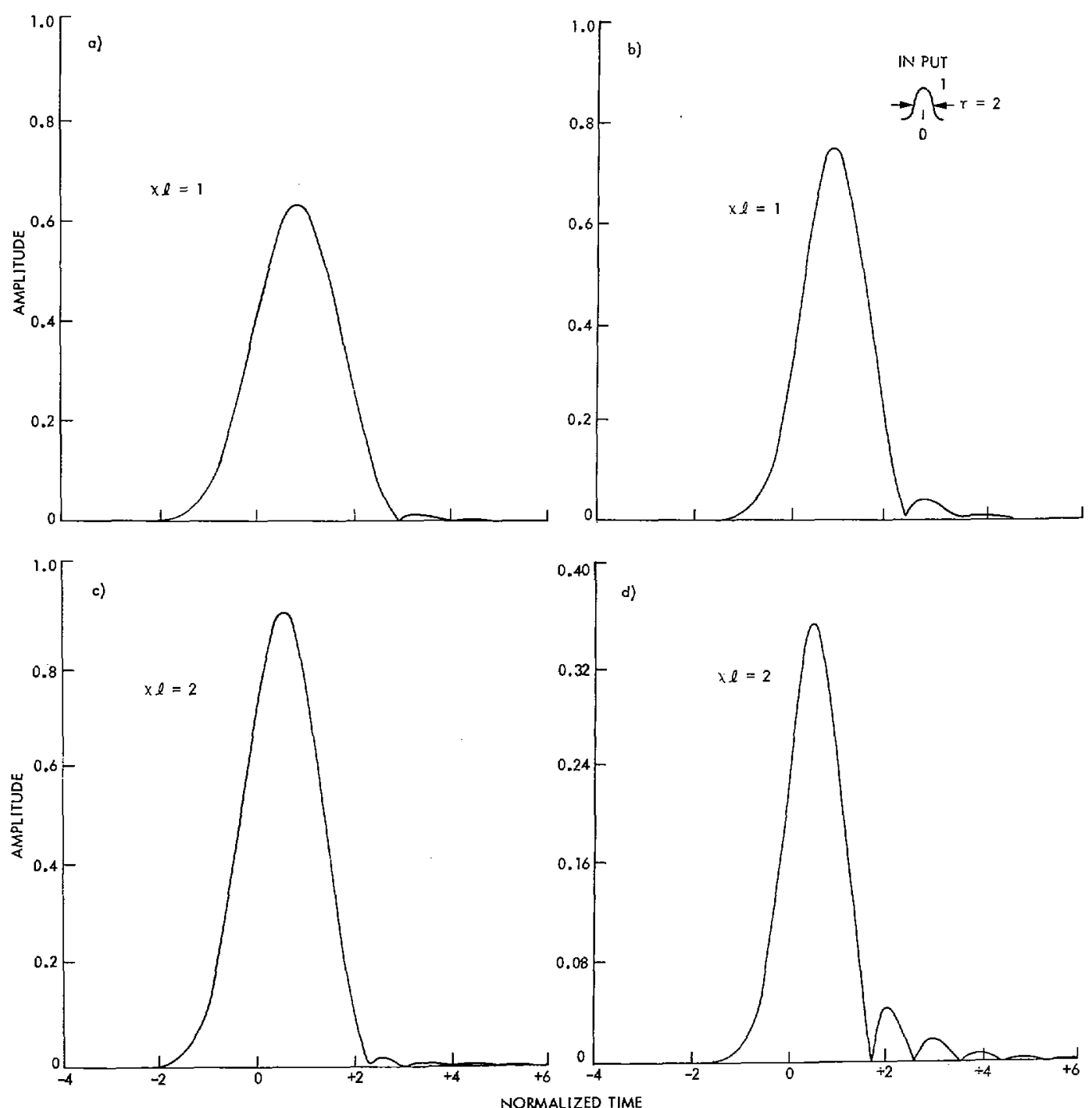

Fig. 6. Reflected and transmitted pulses for case of incident Gaussian pulse of width 2 time units and carrier frequency $\omega_{0}$. Remark difference in vertical scales.

where

$$
D=\left[\chi_{p q}^{2}-\left(\frac{\delta_{p}+\delta_{q}}{2}\right)^{2}\right]^{1 / 2}
$$

If the two coupled modes are identical, then $\delta_{p}=\delta_{q}=\delta$, and

$$
\begin{aligned}
R & =\frac{i \chi}{D \operatorname{coth} D l-i \delta} \\
T & =\frac{D}{D \cosh D l-i \delta \sinh D l} \\
D & =\left(\chi^{2}-\delta^{2}\right)^{1 / 2} .
\end{aligned}
$$

From (6) and (7) we can derive the characteristics of the reflection and transmission coefficients of the periodic structure:

1) maximum reflection

$$
R_{M}=i \tanh \left(\chi_{p q} l\right)
$$

2) minimum transmission

$$
T_{m}=1 / \cosh \left(\chi_{p q} l\right)
$$

3) $R=0$ and $T=1$ for

$$
\delta_{p}+\delta_{q}=2\left[\chi_{p q}{ }^{2}+(n \pi / l)^{2}\right]^{1 / 2}, \quad n=1,2, \cdots
$$

the corresponding phase of $R$ is equal to $(2 n-1) \pi / 2$; 4) $|R|^{2}+|T|^{2}=1$.

In Fig. 2 are shown the magnitudes of the reflection and transmission coefficients given in (8) and (9) as functions of $\delta l$ for a given value of $\chi l$. Fig. 3 shows the equimagnitude and equiphase curves of the reffection coefficient plotted as a function of $\chi l$ and $\delta l$. Fig. 2 corresponds to a horizontal line $(\chi l=2)$ across Fig. 3 . If the two coupled modes are different (i.e., $p \neq q$ ), the curves in Figs. 2 and 3 are still valid if we replace $\delta$ by $\left(\delta_{p}+\delta_{q}\right) / 2$ and $\chi$ by $\chi_{p q}$.

\section{TRANSIENT RESPONSE}

The reflection and transmission of a pulse with a relatively narrow spectrum near $\omega_{p q}$ can now be derived from the 

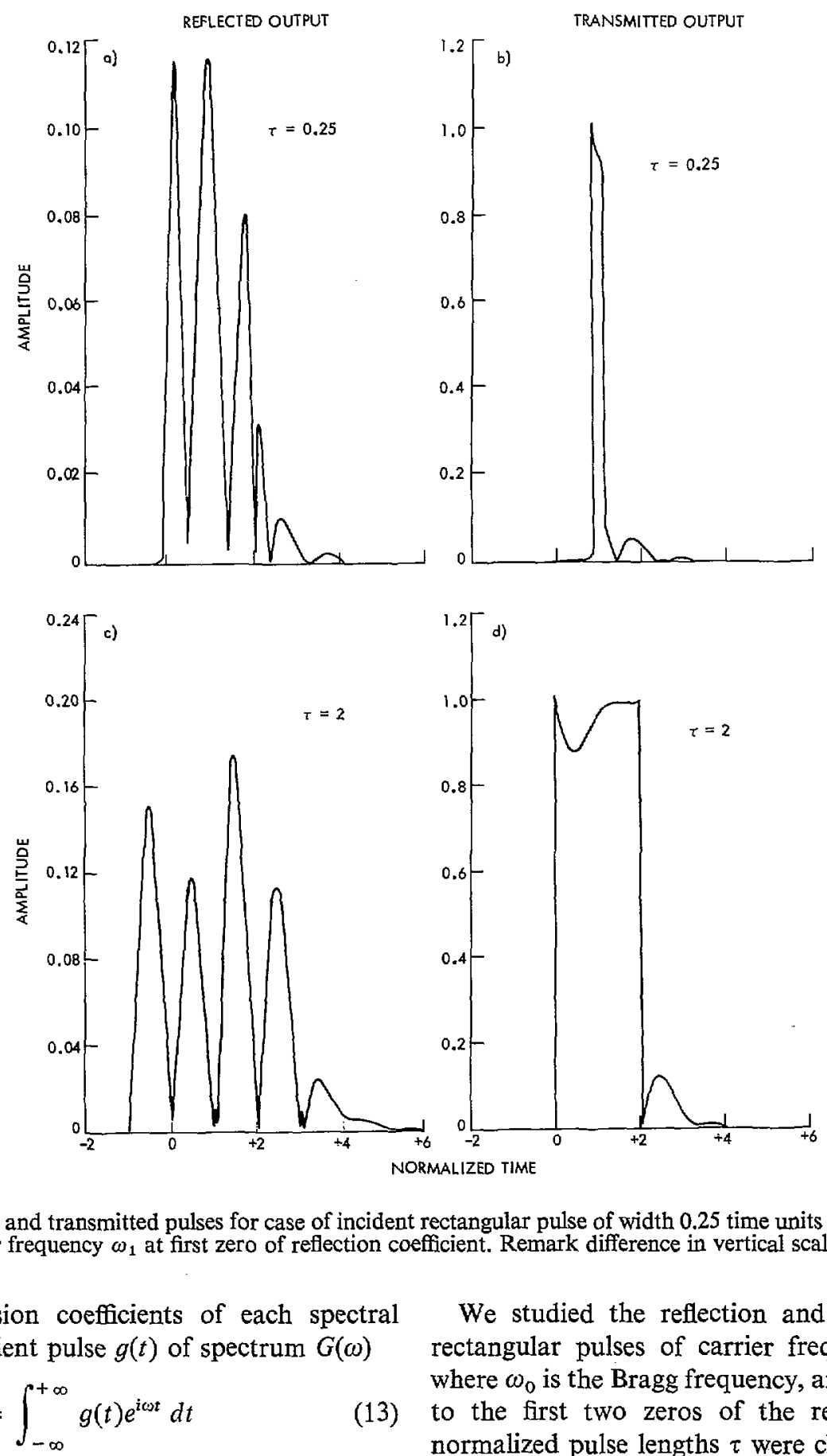

Fig. 7. Reflected and transmitted pulses for case of incident rectangular pulse of width 0.25 time units and 2.0 time units and carrier frequency $\omega_{1}$ at first zero of reflection coefficient. Remark difference in vertical scales. $\chi l=1$.

reflection and transmission coefficients of each spectral component. For an incident pulse $g(t)$ of spectrum $G(\omega)$

$$
G(\omega)=\int_{-\infty}^{+\infty} g(t) e^{i \omega t} d t
$$

the reflected pulse is

$$
r(t)=\frac{1}{2 \pi} \int_{-\infty}^{+\infty} G(\omega) R(\omega) e^{-i \omega t} d \omega
$$

and the transmitted pulse is

$$
p(t)=\frac{1}{2 \pi} \int_{-\infty}^{+\infty} G(\omega) T(\omega) e^{-i \omega t} d \omega .
$$

Numerical inversion has been used by a number of authors $[10],[11]$, [and others] to obtain time response to radiation, propagation, and scattering problems by convolution of transforms. The method used in this paper was the CooleyTukey fast Fourier transform (FFT) [12], with $2^{11}=2048$ samples in the FFT to calculate $r(t)$ and $p(t)$.
We studied the reflection and transmission of several rectangular pulses of carrier frequency $\omega_{0}, \omega_{1}$, and $\omega_{2}$, where $\omega_{0}$ is the Bragg frequency, and $\omega_{1}$ and $\omega_{2}$ correspond to the first two zeros of the reflection coefficient. The normalized pulse lengths $\tau$ were chosen to be 0.25 and 2.0 time units, where each time unit corresponds to the transit time of the pulse across the slab of length $l$.

Fig. 4 displays the envelopes of the reflected and transmitted pulses which result from an incident pulse of length $\tau=0.25$, with a carrier frequency exactly at phase match $\left(\omega=\omega_{0}\right)$. The different cases correspond to different values of the coupling coefficient $\chi$. In all the illustrations, the time reference is such that $t=0$ corresponds to the instant when the center of the incident pulse is at the first boundary. For weak coupling, the reflected pulse is spread over 2.25 time units (Figs. 4(a) and (b)) because the energy is reflected from the successive stratifications and the echo from the last layer would take a round trip time of 2 units. Since the successive reflections are relatively weak $(\chi l \ll 1)$, the reflected pulse is quasi-rectangular, and the transmitted 

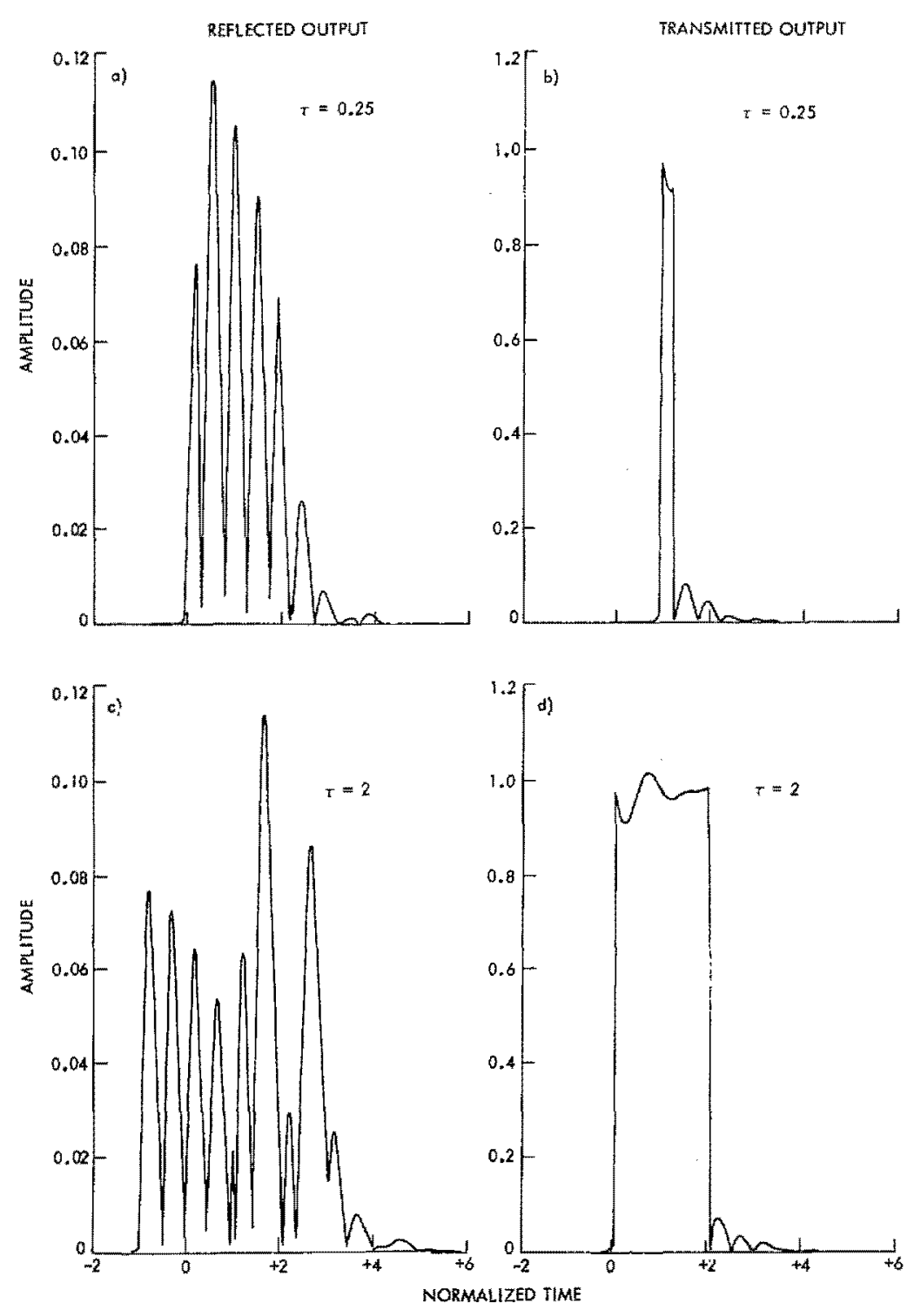

Fig. 8. Reflected and transmitted pulses for case of incident rectangular pulse of width 0.25 time units and 2.0 time units and carrier frequency $\omega_{2}$ at second zero of reflection coefficient. Remark difference in vertical scales. $\not l=1$.

pulse is similar to the incident pulse with a time delay. As the coupling is increased (i.e., stronger reflections from each layer), multiple interference plays an appreciable role which leads to transients over a longer time period. The reflected pulse reaches a maximum at time $t=0.25$ when the whole incident pulse is just inside the slab. The subsequent fall-off in the reflected pulse amplitude is due to the fact that a large portion of the signal has already been reflected back by the first few stratifications. This fall-off is faster for stronger coupling (Figs. 4(e), (g), and (i)). Additionally, a complicated interference tail of multiple subpulses is present. For strong coupling, the transmitted pulse consists mainly of two narrow peaks, 0.25 time units apart, which correspond to differentiation of the incident pulse.

In Fig. 5, the same sequence is repeated for the case of a rectangular pulse of length $\tau=2$. For weak coupling (Fig. $5(\mathrm{a})$ ), the reflected pulse is similar to the autocorrelation of the incident pulse. This is due to the similarity of the input pulse spectrum and the reflection coefficient in the frequency domain, and does not hold for other pulse widths or pulses in general. For strong coupling, the transmitted pulse is again similar to the absolute derivative of the incoming pulse, with the characteristic interference echoes. It should be pointed out that the reflected echo might have an amplitude larger than 1 (Figs. $5(\mathrm{~g})$ and (i)) due to favorable coherent addition of successive reflections.

Fig. 6 displays the reflected and transmitted pulses for a Gaussian incident pulse of width $\tau=2$ (width is taken at the $1 / e$ values). Since a Gaussian pulse spectrum contains smaller high frequency components, the reflected and transmitted pulses are grossly similar to the incident pulse. However, if the incident pulse is narrowed to $\tau=0.25$ (not shown here), the results are somewhat similar to those obtained for a rectangular pulse of the same width.

Finally, a number of curves were generated for the case where the carrier frequency is shifted to the first (Fig. 7) or second (Fig. 8) zero of the reflection coefficient. In these cases, the reflected pulse consists of a number of inter- 
ference pulses which are different from the incident one. We remark that for $\tau=2$, the width of each main pulse is approximately 1 time unit (in Fig. 7) and $\frac{1}{2}$ time unit (in Fig. 8).

\section{Limitations AND APPLiCATIONS}

The preceding results were based on the coupled wave approach which only accounts for the main forward and backward waves and neglects the presence of higher harmonics. This is valid as long as the coupling is weak enough such that the wave amplitude does not change much over a wavelength or similarly over a structure period, and the input bandwidth is not too large. Otherwise the Floquet approach [1]-[5], which accounts for all space-harmonics, has to be used. This approach, even though it is simple to formulate, is relatively involved numerically and is left for future work.

Periodic structures, in different forms, have played an important role in many fields: microwave tubes, filters, antennas, and solid state theory. Recently their application in the emerging field of optical communication and integrated optics [5] has attracted appreciable attention. As it is very likely that optical communication will be in a digital form, the results of this paper are very relevant. For instance, a periodic structure can be used as a differentiator for edge detection (Fig. 5(j)) or multiple pulse generator (Fig. 7). The results can also be applied to many fields like microwave filters, optical filters, and sounders of subsurface layers.

\section{REFERENCES}

[1] N. Brillouin, Wave Propagation in Periodic Structures. New York: Dover, 1953.

[2] T. Tamir, H. C. Wang, and A. A. Oliner, "Wave propagation in sinusoidally stratified dielectric media," IEEE Trans. Microwave Theory Tech., vol. MTT-12, pp. 323-335, May 1964.

[3] K. F. Casey, J. R. Mathis, and C. Yeh, "Wave propagation in sinusoidally stratified plasma," J. Math. Phys., vol. 10, pp. 891$987,1969$.

[4] T. Tamir and H. C. Wang, "Scattering of electromagnetic waves by a sinusoidally stratified half-space," Can. J. Phys., vol. 44, pp. 2073-2093, 1966

[5] C. Elachi and C. Yeh, "Periodic structures in integrated optics," J. Appl. Phys., vol. 44, pp. 3146-3152, 1973.

[6] - "Mode conversion in periodically distributed thin film waveguides," J. Appl. Phys., vol. 45, p. 3494, 1974.

[7] W. H. Louisell, Coupled mode and parametric electronics. New York: Wiley, 1960.

[8] D. Marcuse, Light Transmission Optics. New York: Van Nostrand, 1973.

[9] H. Kogelnik and C. V. Shank, "Coupled wave theory of distributed feedback lasers," J. Appl. Phys., vol. 43, pp. 2327-2335, 1972.

[10] P. S. Ray and J. J. Stephens, "Far-field transient backscattering by ice spheres," Radio Sci., vol. 9, pp. 43-55, 1974.

[11] D. A. Hill and J. R. Wait, "The transient electromagnetic response of a spherical shell of arbitrary thickness," Radio Sci., vol. 7, pp. 911-935, 1972.

[12] J. W. Cooley, P. A. Lewis and P. D. Welch, The Fast Fourier Transform Algorithm. Yorktown Heights, N.Y.: IBM Watson Research Center, 1969.

\title{
Detection and Discrimination of Radar Targets
}

\author{
DAVID L. MOFFATT AND RICHARD K. MAINS
}

\begin{abstract}
A new method for detection and discrimination of radar targets is described. The basis for this method is that the gross structure of a radar target can be identified from scattered fields of the target at harmonic radar frequencies located just in the low resonance region. This is in sharp contrast to many target signature schemes that operate at much higher frequencies and obserye many of the details of the target in lieu of its gross features. Multiple frequency radar scattering data and the complex natural resonant frequencies of radar targets are integrated into a predictor-correlator processor. The method is illustrated using as target models both classical shapes and thin-wire configurations of simple geometry. Integral equation programs are utilized to calculate multiple frequency backscatter data for the wire geometries and to deduce the complex natural resonant frequencies of the wire structures. Discrete
\end{abstract}

Manuscript received July 30, 1974; revised December 29, 1974. This work was supported in part by Contract F-19628-72-C-0203 between Air Force Cambridge Research Laboratories, Laurence G. Hanscom Field, Bedford, Mass., and the Ohio State University Research Foundation, Columbus, Ohio.

D. L. Moffatt is with the Ohio State University ElectroScience Laboratory, Department of Electrical Engineering, Columbus, Ohio 43212 .

R. K. Mains was with the Ohio State University ElectroScience Laboratory, Department of Electrical Engineering, Columbus, Ohio. He is now with the University of Michigan, Ann Arbor, Mich. multiple frequency radar scattering data spanning a particular spectral range are shown to be desirable for optimum capability but discrimination and detection can be achieved using just two near-conventional radars, even if the radars are located at different sites and hence view the target from different aspects.

\section{INTRODUCTION}

T HIS PAPER HAS its basis in the "impulse response" concept advanced by Kennaugh and Cosgriff in 1958 [1] and formalized by Kennaugh and Moffatt in 1965 [2]. The latter paper contained two results of paramount importance to target detection and discrimination studies. First, it was demonstrated that discrete spectral scattering data spanning the Rayleigh and low resonance ranges of the scatterer defined the gross physical properties (size, shape, and composition) of the target. Second, it was noted that the transient response waveform defined by Rayleigh and low resonance range data, the ramp response waveform, appeared easily amenable (for all but short times) to approximations by finite exponential sums. Thus rational function approximations were postulated for the scattering 